\title{
APPLICATION OF THERMOELECTRIC POWER MEASUREMENTS TO THE STUDY OF COLD ROLLED AUSTENITIC STAINLESS STEELS
}

\author{
C. Capdevila, T. De Cock, F. G. Caballero, D. San Martin and C. Garcia de Andres
}

MATERALIA Group, Department of Physical Metallurgy, Centro Nacional de Investigaciones Metalúrgicas (CENIM), Consejo Superior de Investigaciones Científicas (CSIC), Avda. Gregorio del Amo, 8, E-28040 Madrid, Spain.

\begin{abstract}
In this work, it is demonstrated that thermoelectric power measurements constitute a very useful tool to monitor the recrystallised grain growth evolution in cold rolled austenitic stainless steel with reductions varying between $30 \%$ and $80 \%$, and annealed at different temperatures.
\end{abstract}

Keywords: AISI 304, Recrystallisation, Grain Growth, Thermoelectrical power measurement 


\section{Introduction}

Austenitic stainless steels AISI 304 are widely used steels combining high corrosion-oxidation resistance, good heat resisting properties and a relative low cost. In recent years an increasing interest can be noted in the development of new processing routes, for which a detailed study of the microstructural evolution of the cold-rolling material at different annealing temperatures is required. Due to the complex nature of these materials, a technique that allows for a precise monitoring of the grain size evolution at different annealing stages was lacking up to now.

In this context, the aim of this work is to evaluate the utility of the thermoelectic power (TEP) measurements to characterise the recrystallised grain growth of the AISI 304 steels annealed at temperatures between 1000 and $1200{ }^{\circ} \mathrm{C}$ and for different rolling reductions. Several authors [13] reported that this technique is very sensitive to both the amount of atoms in solid solutions (i.e. precipitation and dissolution processes) and to the dislocation state of the material (i.e. deformation and recovery). J.P. Ferrer et al. [4] applied this knowledge to carry out a detailed study of the annealing behaviour in cold and warm rolled ELC steels. These studies suggest that TEP measurements could be a very interesting technique for the qualitative and quantitative study of the recrystallised grain size, in the cases where no other phenomena, distorting the global TEP signal, take place and taking into account that such effects as crystallographic orientation, grain size distribution or grain boundary structure can not be observed by this technique.

\section{Materials and experimental techniques}

The chemical composition of the studied steel in wt $\%$ is as follows: $0.039 \% \mathrm{C}, 0.22 \% \mathrm{Si}$, $0.001 \% \mathrm{~S}, 0.029 \% \mathrm{P}, 1.15 \% \mathrm{Mn}, 18.0 \% \mathrm{Cr}, 8.52 \% \mathrm{Ni}, 0.24 \% \mathrm{Mo}, 0.25 \% \mathrm{Cu}, 0.0005 \% \mathrm{~B}$, and $0.04 \% \mathrm{~N}$. The material was obtained from a hot-rolled coil and was subsequently cold rolled down to $30 \%, 70 \%$ and $80 \%$ reduction. In order to study the evolution of the recrystallised grain growth, samples $30 \mathrm{~mm}$ in length, $2 \mathrm{~mm}$ in width and $0.8 \mathrm{~mm}$ in thickness were annealed at different temperatures $\left(1000,1050,1100,1150\right.$ and $\left.1200{ }^{\circ} \mathrm{C}\right)$. After 180 seconds of holding time, specimens were rapidly cooled to room temperature and the corresponding TEP value was measured on these samples immediately afterwards. These heat treatments were appropriately chosen in order to obtain fully recrystallised samples in each case.

A schematic representation of the TEP apparatus is given elsewhere [5-7]. The experimental procedure of the TEP measurement is the following: the sample is pressed between two blocks of 
a reference metal (in this case, pure copper). One of the blocks is at $15^{\circ} \mathrm{C}$, while the other is at $25{ }^{\circ} \mathrm{C}$ to obtain a temperature difference $\Delta \mathrm{T}$. A potential difference $\Delta \mathrm{V}$ is generated at the reference metal contacts. The apparatus does not give the absolute TEP value of the sample $\left(S^{*}\right)$, but a relative TEP $(S)$ in comparison to the TEP of pure copper $\left(S_{0}{ }^{*}\right)$ at $20{ }^{\circ} \mathrm{C} . S$ is given by $\mathrm{S}=\mathrm{S}^{*}-\mathrm{S}_{0}^{*}=\Delta \mathrm{V} / \Delta \mathrm{T}$. The TEP value does not depend on the shape of the sample as long as the ratio between section $(\mathrm{S})$ and length $(\mathrm{L})$ is lower that $\mathrm{S} / \mathrm{L}<0.5(\mathrm{~mm})$, which for the case of study is fulfilled, i.e. $\mathrm{S} / \mathrm{L}=0.053(\mathrm{~mm})$. Moreover, the measurement is performed very quickly (less than $1 \mathrm{~min}$ ) and precisely (about $\pm 0.5 \%$ ). The resolution is of the order of $1 \mathrm{nV} / \mathrm{K}$ and the typical error is below $10 \mathrm{nV} / \mathrm{K}$.

The samples were etched with a solution containing 60\% hydrochloric acid, 20\% nitric acid and $20 \%$ methanol to reveal the individual grains. The presence of second phases in the microstructure was disclosed by means of the Lichtenegger-Bloch reagent [8,9] which consists of $20 \mathrm{~g}$ ammonium difluoride, $0.5 \mathrm{~g}$ potassium bisulfite and $100 \mathrm{ml}$ hot distilled water.

\section{Results and discussion}

Figure 1 shows the optical micrographs of the annealed microstructures with increasing annealing temperature for the case of $30 \%$ deformation. The applied etching procedure enables to reveal the grain size, which was measured on optical micrographs by standarised image analysis techniques. The evolution of the recrystallised grain size (expressed as the average grain diameter) with the annealing temperature, which has been measured for each degree of deformation is shown in Fig. 2.

Subsequently, for each annealing stage the corresponding TEP value was measured, taking the TEP value at the lowest annealing stage (i.e. at $1000^{\circ} \mathrm{C}$ ) as a reference level for each deformation level. The results of the TEP analysis is given in Figure 3.

From comparison between Fig. 2 and 3, it can be clearly seen two different behaviours in the evolution of TEP and grain diameter. The higher deformation of the material (70 and 80\%) leads to higher recrystallised grain sizes and higher values of TEP compared to the case of small deformation (30\%). Whereas a clear influence of high temperature can be noted on TEP values of high deformed samples, the negative values of the TEP at lower holding temperatures and at lower deformation degrees of deformation indicate that the TEP measurement in these cases is dominated by other effects, e.g. dissolution processes and generation of vacancies during annealing. 
The presence of delta ferrite as a second phase in AISI 304 has been broadly reported before [10]. To study the possible influence of a second phase, the samples were etched with the Lichtenegger-Bloch reagent. As can be seen in Fig. 4, the cold-rolled steel with $30 \%$ reduction contains delta ferrite phase, which is known to constitute a preferential nucleation site for $\mathrm{M}_{23} \mathrm{C}_{6}$ particles [11], primarily at the $\delta / \gamma$ interface. Since during annealing at high temperatures these particles dissolve very rapidly, the solute content of elements such as $\mathrm{C}$ and $\mathrm{Mn}$ increases, which explains the negative TEP values. At high deformation levels, no delta ferrite was detected, and thus the dissolution mechanism does not apply in this case, leading to high TEP values. The behaviour of the steels with deformation degrees of 70 and $80 \%$ is very similar, except for low and high annealing temperatures: at low annealing temperatures a small influence of dissolution processes in the steel with cold rolling degree of $70 \%$ is observed, which is not the case for the steel with $80 \%$ reduction. This suggests that a treshold value exists for the TEP sensitivity regarding the grain size, lying around $50 \mu \mathrm{m}$ in the present case, above which the TEP values are dominated by the grain size effect. It can be seen that for the low deformation steel $(30 \%)$ this value is not reached until the annealing stage at $1150^{\circ} \mathrm{C}$, resulting in low TEP values at earlier stages due to the dissolution effect.

On the other hand, at high annealing temperatures the average recrystallised grain size of the steels with $70 \%$ reduction is slightly higher than for steels with $80 \%$ reduction. This decrease of the grain growth evolution in the steel with $80 \%$ reduction is also reflected by the lower TEP signal measured (Fig. 3), which is another demonstration of the utility of this technique.

When comparing the evolution of the recrystallised grain size and thermoelectric power measurements for each cold rolling degree (Fig. 5), it can be clearly seen that in the range of grain sizes studied a linear relation exists between both variables, except for the low annealing temperatures, where dissolution processes alter the TEP measurements. After these initial values, a perfect linear correlation between grain size and thermoelectric power is observed, allowing for a precise prediction of the grain size in function of TEP. It can be assumed that a grain boundary is an agglomeration of 2-dimensional defects such as grain boundary dislocations. Therefore, the observed increase in TEP is caused by the annihilation of GB dislocations during the grain growth process.

It could be concluded from the results presented that the increase of the grain size is still relatively limited at lower annealing stages. At these annealing stages, the contribution to the TEP measurements is dominated by the dissolution of particles. By contrast, at higher annealing temperatures and higher deformations, the coarsening of recrystallised grain is the main contribution to TEP. These observations can be observed with high precision by means of 
thermoelectric power measurements, which demonstrates the utility of this novel technique for the study of recrystallisation and dissolution processes. Thus, TEP could be a very interesting tool for in-situ studies of the recrystallized grain size during annealing, even in steels where the metallographic analysis is not straightforward. In view of this, the work lays the foundation for future in-situ measurements, since it establishes an unambiguous relation between the TEP signal and the average recrystallized grain size after annealing as determined by optical microscopy. However, TEP is also sensitive to microstructural changes as a result of dissolution phenomena that can take place simultaneously with recrystallisation. Particularly at low annealing stages, the contribution of grain growth to the TEP signal is distorted by the influence of these phenomena, leading to a non-linear behaviour between recrystallised grain size and TEP. Therefore, TEPmeasurements should be interpreted with great care.

\section{Conclusions}

At low annealing temperatures, the contribution to the TEP measurements is dominated by the dissolution of particles. By contrast, at higher annealing temperatures and higher deformations, the coarsening of recrystallised grain is the main contribution to TEP, which induce straight line dependence. These observations demonstrate the utility of this novel technique for the study of recrystallisation and dissolution processes.

\section{Acknowledgements}

The authors acknowledge financial support from European Coal and Steel Community (ECSC7210-PR-368) and from Spanish Ministerio de Educación (MAT2002-18810-E). T. De Cock would also like to express his gratitude to the CSIC for financial support in the form of a $\mathrm{PhD}$ research grant (I3P program). The authors also acknowledge Javier Vara for the experimental support.

\section{References}

[1] N. LAVAIRE, V. MASSARDIER and J. MERLIN, Scr. Mater. 50 (2004) 131.

[2] H. ABE, Scand. J. Metall. 13 (1984) 226.

[3] BRAHMI and R. BORRELLY, Acta Mater. 45 (1997) 1889.

[4] J.P. FERRER, T. DE COCK, C. CAPDEVILA, F.G. CABALLERO and C. GARCÍA DE ANDRÉS, Acta Mater. 55 (2007) 2075.

[5] G. VANDER VOORT, in "Metallography Principles and Practice" (ASM International, New York, 1999). 
[6] CAPDEVILA, F.G. CABALLERO and C. GARCIA DE ANDRES, Mat. Sci. Technol. 19 (2003) 581.

[7] F.G. CABAllero, A. GARCÍA-JUNCEDA, C. CAPDEVIlA and C. GARCÍA DE ANDRÉS, Scr. Mater. 52 (2005) 501.

[8] SAN MARTIN, P.E.J. RIVERA DIAZ DEL CASTILLO, E. PEEKSTOK and S. VAN DER ZWAAG, Mater. Charact. 58 (2007) 455-460.

[9] N.J. LUIGGI, Metall. Mater. Trans. A 29 (1998) 2669.

[10] K. BORST and M. POHL, Steel Research 61 (1990) 258.

[11] S.H. KIM, H.K. MOON, T. KANG and C.S. LEE, Mat. Sci. Eng. A356 (2003) 390. 


\section{Figure Captions}

Figure 1. Optical micrographs of the AISI 304 steel with $30 \%$ rolling reduction (a) in the as-cold rolled condition and after heat treatment at (b) $1000{ }^{\circ} \mathrm{C}$, (c) $1050{ }^{\circ} \mathrm{C}$, (d) $1100{ }^{\circ} \mathrm{C}$, (e) $1150{ }^{\circ} \mathrm{C}$ and (f) $1200^{\circ} \mathrm{C}$.

Figure 2. Evolution of the recrystallised grain size for the three cold rolling reductions

Figure 3. Evolution of the thermoelectric power for the three cold rolling reductions.

Figure 4. Lichtenegger-Bloch etch of the sample deformed at $30 \%$ and after annealing at $1000^{\circ} \mathrm{C}$. The white phase corresponds to delta ferrite, the brown phase is the austenitic matrix.

Figure 5. Relation between the thermoelectric power and recrystallised grain size for the three cold rolling reductions. 


\section{Figures}
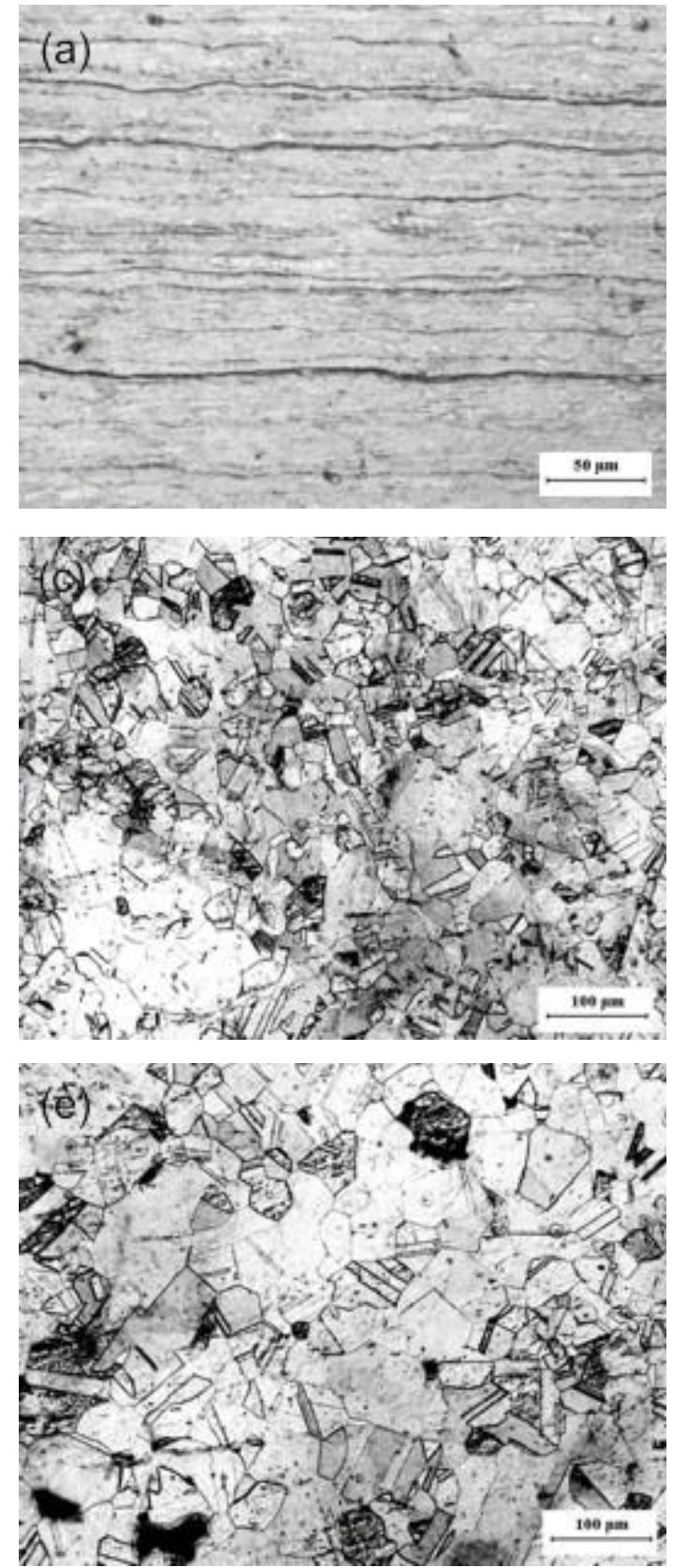
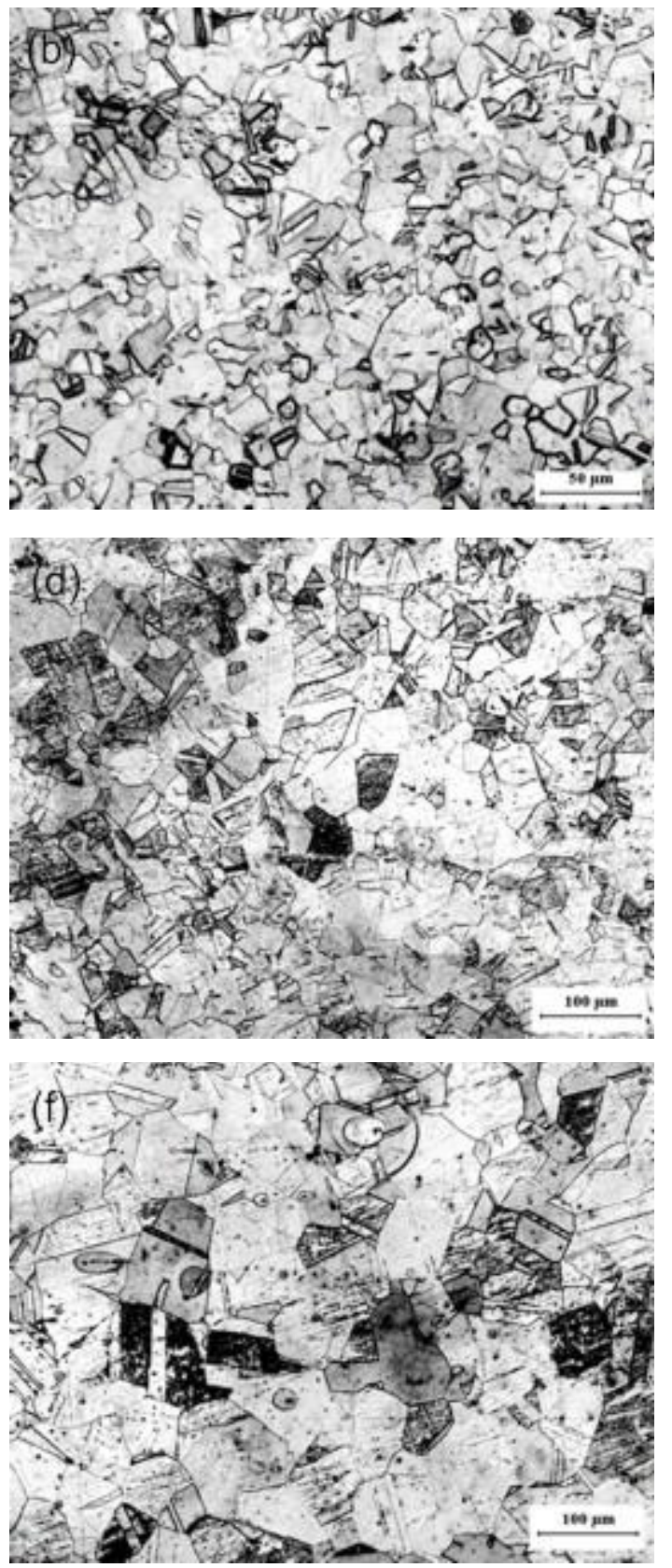

Figure 1. Optical micrographs of the AISI 304 steel with $30 \%$ rolling reduction (a) in the ascold rolled condition and after heat treatment at (b) $1000^{\circ} \mathrm{C}$, (c) $1050{ }^{\circ} \mathrm{C}$, (d) $1100{ }^{\circ} \mathrm{C}$, (e) 1150 ${ }^{\circ} \mathrm{C}$ and (f) $1200{ }^{\circ} \mathrm{C}$. 


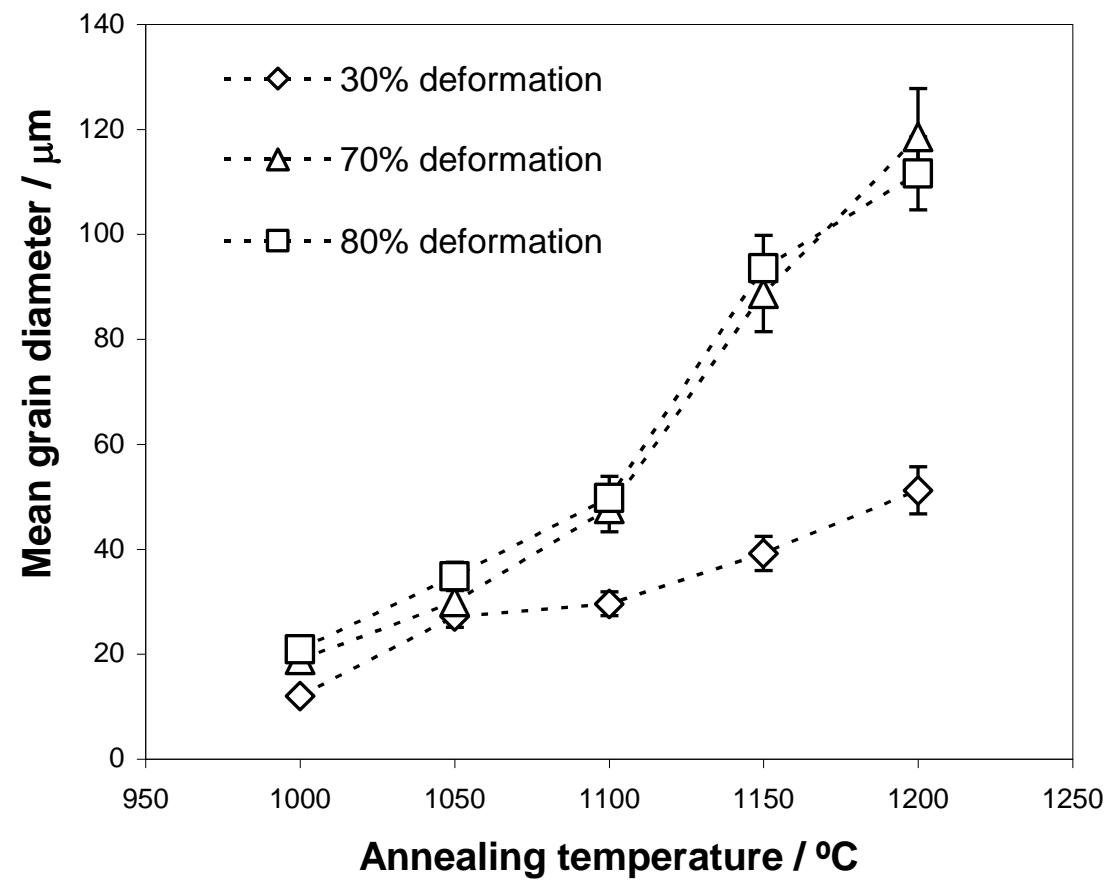

Figure 2. Evolution of the recrystallised grain size for the three cold rolling reductions 


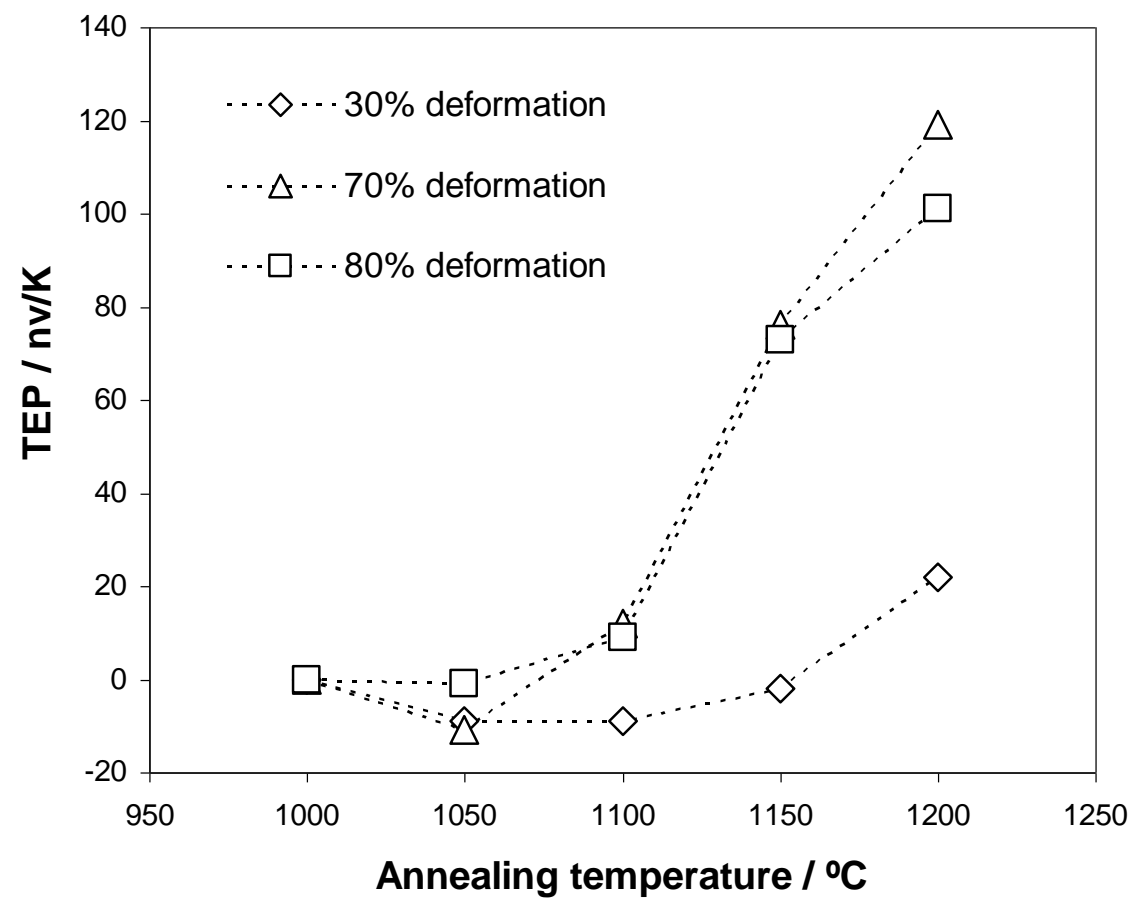

Figure 3. Evolution of the thermoelectric power for the three cold rolling reductions. 


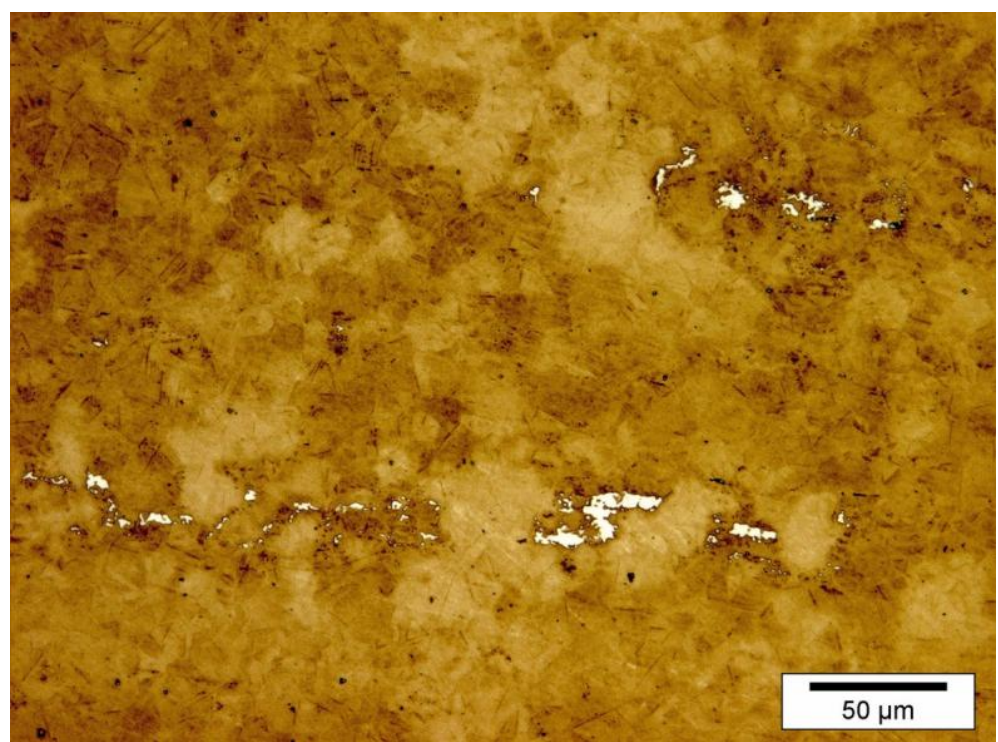

Figure 4. Lichtenegger-Bloch etch of the sample deformed at $30 \%$ and after annealing at $1000^{\circ} \mathrm{C}$. The white phase corresponds to delta ferrite, the brown phase is the austenitic matrix. 


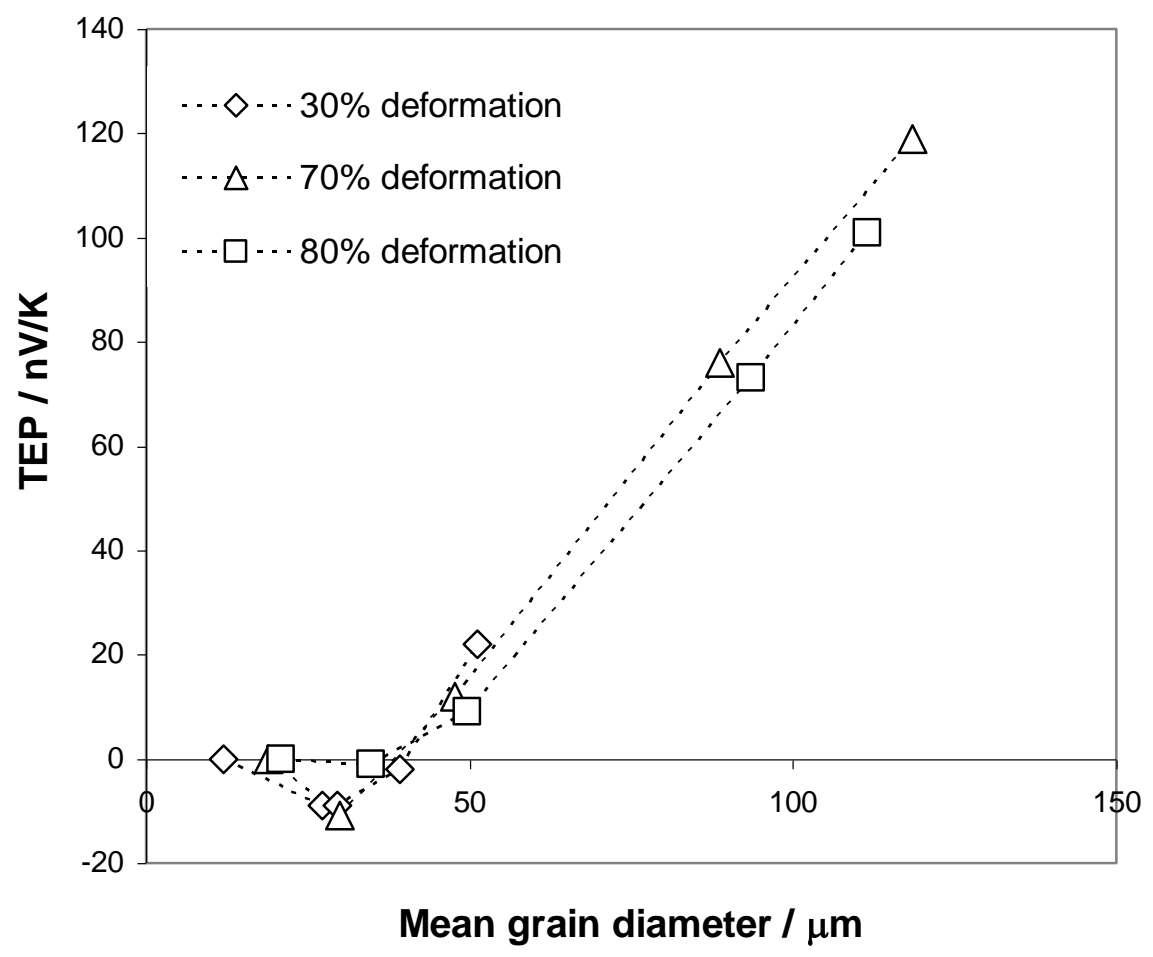

Figure 5. Relation between the thermoelectric power and recrystallised grain size for the three cold rolling reductions. 